\title{
THE HISTORY OF THE DEVELOPMENT OF THE HEXAMETER IN GERMAN POETRY
}

\author{
Tatiana N. Andreiushkina \\ Togliatti State University (Togliatti, Russia) \\ ORCID ID: http://orcid.org/0000-0003-2818-983X
}

\begin{abstract}
A bstract. The article traces the history of the development of the hexameter on German soil: from the use of the Leonin hexameter in the Middle Ages and the Reformation, the mixed Latin-German hexameter in the period of humanism (in the form of carmina eroica) and the German hexameter in the $18^{\text {th }}-19^{\text {th }}$ centuries (mainly in the form of elegy, epigram and idyll) to derivatives and ironic forms of the XX century (memorandum, instructive poem, etc.). Klopstock played a significant role in the spread of the hexameter in German poetry, bringing a fresh stream to German poetry by rejecting the prevailing in the $17^{\text {th }}$ century predominantly alternating Alexandrian verse. Voss also inspired his contemporaries to create distiches with his translations of Homer's poems. The flowering of the hexameter falls on the period of classicism: Goethe and Schiller created the best and purest examples of this poetic meter. Goethe and Schiller during the Enlightenment, Hölderlin, Novalis and Kleist in romanticism, Rückert, Platen and Mörike in post-romanticism introduced variety and movement into the hexameter by means of different types of caesura in verse. Austrian poets (Saar, Weinheber, Bachmann) appeal to hexameter as a classic form of German verse, Hauptmann uses it to create a large poetic form. The poets of the pre-war and war period (Colmar, Schröder, Holthusen) seek in him an aesthetic support in an era of timelessness. Poets of the former GDR (Brecht, Bobrowski, Müller), poets of the Federal Republic of Germany (Grünbein, Herbst) use it sporadically and in a transformed form, but at the same time take into account the thematic and genre traditions associated with this antique meter. Most foreign researchers, when determining the hexameter, speak of its dactylic component and only from the middle of the 2oth century some of them (Kayser, Mönnighof) note, in addition to the spondees, the possibility of using chorees in the initial syllables of a verse.
\end{abstract}

Ke ywords: hexameter; Leonine verse; elegiac distich; idyll; epigram; didactic poem.

\section{ИСТОРИЯ РАЗВИТИЯ ГЕКЗАМЕТРА В НЕМЕЦКОЯЗЫЧНОЙ ПОЭЗИИ}

\author{
Андреюшкина Т. Н. \\ Тольяттинский государственный университет (Тольятти, Россия) \\ ORCID ID: http://orcid.org/0000-0003-2818-983X
}

Анно таиия. В статье прослеживается история развития гекзаметра на немецкой почве: от употребления леонинского гекзаметра в Средневековье и Реформации, смешанного латинско-немецкого гекзаметра в период гуманизма (в виде carmina eroica) и немецкого гекзаметра в XVIII-XIX вв. (преимущественно в форме элегии, эпиграммы и идиллии) до дериватов и иронических форм XX в. (меморандум, поучительное стихотворение и др.). Значительную роль в распространении гекзаметра в немецкой поэзии сыграл Клопшток, внеся свежую струю в немецкую поэзию отказом от преобладавшего в XVII в. преимущественно альтернирующего александрийского стиха. Фосс своими переводами поэм Гомера также вдохновил современников на создание дистихов. Расцвет гекзаметра приходится на период классицизма: Гете и Шиллер создали лучшие и чистые образцы этого стихотворного размера. Гете и Шиллер в период Просвещения, Гельдерлин, Новалис и Клейст в романтизме, Рюкерт, Платен и Мерике в постромантизме вносили разнообразие и движение в гекзаметр путем различного вида цезур в стихе. К гекзаметру как классическому виду немецкого стиха апеллируют австрийские поэты (Заар, Вайнхебер, Бахман), Гауптман использует его для создания крупной стихотворной формы. Поэты предвоенной и военной поры (Кольмар, Шредер, Хольтхузен) ищут в нем эстетическую опору в эпоху безвременья. Поэты бывшей ГдР (Брехт, Бобровский, Мюллер), поэты ФРГ (Грюнбайн, Хербст) используют его спорадически и в трансформированной форме, однако при этом учитывают тематические и жанровые традиции, связанные с этим античным размером. Большинство зарубежных исследователей при определении гекзаметра говорят 
о его дактилической составляющей, и только с середины XX в. некоторые из них (Кайзер, Мёнигхоф) отмечают помимо спондеев возможность применения хореев в начальных тактах стиха.

Ключев в е слов а : гекзаметр; леонинский стих; элегический дистих; идиллия; эпиграмма; поучительное стихотворение.

Для иитирования:Андреюшкина, Т. Н. История развития гекзаметра в немецкоязычной поэзии / Т. Н. Андреюшкина. - Текст: непосредственный // Филологический класс. - 2021. - Т. 26, № 1. C. 22-37. - DOI: 10.51762/1FK-2021-26-01-02

Einleitung. Zum Thema des Artikels wird die Herausarbeitung des antiken Versmaßes, das eine reiche Tradition nicht nur in der Geschichte der antiken Kunst, sondern auch der europäischen Dichtung gespielt hat, auf deutschem Boden. Die Behandlung dieses Themas ist aufs Engste mit dem aktuellen Problem des Gedächtnisses und des Kanons in der Literatur verbunden, was auf die Aktualität des Artikels hinweist. Der antike Vers wurde in den wissenschaftlichen Werken von deutschen [Kelletat 1964; Arnst 1984; Kayser 1999; Breuer 1999; Knörrich 2005] und russischen Gelehrten untersucht [Tomaschewski 2001, Cholschewnikow 2002, Gasparow 1989, 1999 u. a.]. In folgenden Studien wurden einzelne Probleme des deutschen Verses und auch mancher in Hexametern abgefassten Genres (darunter Elegie, Epigramm, Idylle) zur Sprache gebracht [Bunge 1963; Wagenknecht 1981; Behrmann 1989; Beissner 1991; Moennighoff 2004]. Es wurden auch einzelne in Hexametern geschriebene Werke der deutschen Autoren interpretiert [Freund 1990; Kayser 1991; Suvin 2008]. Aber es gibt keine übersichtliche, geschichtsbezogene analytische Betrachtung der Entwicklung des hexametrischen Verses auf deutschem Boden vom Mittelalter bis heute. Der Artikel begleicht diesen Mangel. Das Ziel dieses Artikels besteht darin, eine kurze, aber systematisch abgefasste Geschichte des Hexameters in der deutschen Dichtung mit mehreren Textzitaten zu präsentieren.

\section{Entstehung des Hexameters als leoninischen} Verses im Mittelalter. Die Geschichte des Hexameters in Deutschland beginnt im 9. Jahrhundert und tritt in Form des leoninischen Hexameters auf. Möglicherweise ist der leoninische Hexameter aus der klassischen römischen Dichtung
For citation: Andreiushkina, T. N. (2021). The History of the Development of the Hexameter in German Poetry. In Philological Class. Vol. 26. No. 1, pp. 2237. DOI: $10.51762 / 1$ FK-2021-26-01-02

(Ovid), aus dem auch auf zwei Silben reimenden Pentameter entstanden, so Knörrich [Knörrich 2005: 132].

Der leoninische Hexameter kam vor allem in der späten antiken und frühmittelalterlichen lateinischen Dichtung vor und diente als mögliches Muster für die Reimgedichte von Otfried von Weißenburg (ca. 800-871), der im Vorwort zum Evangelienbuch (863-871) unter dem lateinischen Titel „Cur scriptor hunc librum theotisce dictaverit“ („Warum der Author in der deutschen Sprache sprechen will“) erklärte, dass er wie andere Dichter in der Muttersprache schreiben will. Der Anvers wird im leoninischen Vers in der Regel auf den Abvers gereimt.

Nist si so gisungan, mit gerulu bithwungan:

Si habet thoh thia rihti in sconeru slihti.

Ili thu zi note, theiz scono thoh gilute,

Joh gotes wizod thanne tharana scono helle

[Otfried 1965: 9]

(Ist sie auch nicht so gesungen (im Gesang gepflegt), / durch (metrische) Regel gebändigt, / so hat sie doch (die) Geradheit in schöner Einfachheit. / Trachte du in notwendiger Weise (beharrlich), / dass es dennoch schön erklinge / und Gottes Gesetz dann / darin schön ertöne) [ibidem].

Führen wir noch einen leoninischen Vers als Epigramm aus dem 9. / 10. Jahrhundert eines Unbekannten Verfassers:

liubene ersatza sine gruz unde kab sina tohter uz. To cham aber starzfidere, prahta imo sina tohter uuidere

[DL 2001, 1: 39].

(Liubene setzte sein Weizenbier an und ließ seine Tochter heiraten.

Da kam strazfidere wieder, brachte ihm seine Tochter zurück) [ibidem].

Noker von Zwiefalten (gest. 1095) setzt die gegebenen Versuche in 19 Strophen seines Ge- 
dichtes „Memento mori“ (1070) mit den Vierzeilern fort und verwendet ähnliche Reime, wie Otfried. Er bildet die Reime, wenn einer davon aus zwei Wörtern besteht (mundus - uns sus), und schließt auch lateinische Wendungen in den Vers ein (nobis miserere) [DL 2001, 1: 142].

Oft wird in den Verspoetiken der spätere leoninische Vers aus Archipoeta (ca. 1130-1165) angeführt, noch auf Latein geschrieben, in dem Kurzverse aufeinander gereimt werden:

En habeo versus te praecipiente reversus sit tibi fons laeta versus recitante poeta

[zit. nach: Knörrich 2005: 132].

(Heimgekehrt entsprechend deinem Wunsch, bringe ich Verse mit.

Möge deine Stirn sich erhellen, wenn der Dichter sie vorträgt) [ibidem].

Die Blütezeit des leoninischen Verses fällt ins 10. - 11. Jahrhundert. In der deutschen Dichtung kam er später auch bei Eberhard von Cersne, Johannes Rothe, zur Zeit des Humanismus bei J. Fischart (1546-1590) vor, als sich neolateinische Dichtung verbreitete, und sogar beim Postromantiker Fr. Rückert, der verschiedene klassische Genres und Metren pflegte.

Lateinisch-deutsche Hexameter: Übergangsformen im Humanismus. Meistersinger S. Brandt hat 1492 ein episches Gedicht „Uon dem donnerstein gefallen im xcij. iar vor Ensishein" geschrieben. Den 22 lateinischen Distichen steht in der rechten Spalte des Flugblattes eine deutsche Parallelfassung gegenüber, die wir als Übersetzung nach dem lateinischen Textauszug anbieten. Der deutsche Text entspricht dem lateinischen im Großen und Ganzen - es fehlen ihm dennoch der Vergleich mit dem Planeten Saturn (35. Zeile), der Hinweis auf Anaxagoras und die Schwierigkeiten einer naturwissenschaftlichen Erklärung (Zeilen 40-43). Vielleicht wollte Brandt im deutschen Text nicht auf Details eingehen. Im Vergleich zu dem lateinischen wird der deutsche Text mit paarweise gereimten Knittelversen abgefasst, deswegen trifft man darin außer Inversionen auch Elisionen, was dem Vers einen umgangssprachlichen Stil verleiht.

Perlegat antiquis miracula facta sub annes

Qui volet: et nostros comparet inde dies.

Uise licet fuerint portenta / horrendaque monstra Lucere e celo: flamma / corona / trabes

[DL 2001, 2: 385].
Sich wundert mancher fremder gschicht

Der merck vnd leß ouch diß bericht

Es sind gesehen wunder vil

Jim lufft comet vnd furen pfil [ibid.: 386].

Mit gleichen Verspaaren (insgesamt 13) wird der Dialog „De periculoso scacorum ludo: Inter mortem et humanam conditionem“ („Über das gefahrvolle Schachspiel zwischen dem Tod und dem Menschen") geschrieben, wo der Tod bekennt, dass ihm alle gehorchen, und zum Schluss erzählt er ein Rätsel über den Baum, der die Zeit verkörpert [DL 2001, 2: 402]. Die Besonderheit dieses Gedichtes besteht darin, dass mit lateinischen Hexametern geschriebene Paare durch die deutschen vierhebigen Reimpaare abgelöst und gemischte lateinisch-deutsche Strophen gebildet werden. Dadurch kommt Brandt zu der Schöpfung des deutschen Hexameters sehr nah.

Noch näher bringt ein Unbekannter Verfasser sein „Carmen ad Clerikum“ („Gedicht an den Klerus“, 1505) dem deutschen Hexameter, während er die linke Hälfte des Gedichtes auf Latein und die rechte Hälfte auf Deutsch gedichtet hat.

Et vt cognoscas latius / So nym gar eben war Ludimagister fatuus / Das ist weyt offenbar

[DL 2001, 3: 23].

Knörrich hat teilweise recht, wenn er meint, dass erst im Humanismus viel Mühe gegeben wird, um den Hexameter deutsch klingen zu lassen, zuerst auf dem Grund des quantitativen Prinzips [Knörrich 2005: 94].

Mehrere lyrische Genres wurden mit lateinischen Distichen geschrieben, aber sie warfen Probleme der deutschen Gesellschaft auf, z. B. das Lehrgedicht von Conrad Celtis „Ad Iuuentutem Germanicam“ („An die deutschen Jugendlichen", 1502) [DL 2001: 3, 19], ein Vermächtnis des Dichters. Ein anderes Beispiel - „Pro sedanda peste ad divam die genitricem elegidion“ („,Kleine Elegie an die heilige Mutter Gottes zur Eindämmung der Pest (Syphilis)“, 1504) [DL 2001, 3: 22]) findet einen Widerhall in der Elegie von U. von Hutten „Obsessus a Gallis cum salutem desperasset“ („Als er hart bedrängt durch die Franzosen (die Syphilis) "), 1538), wo dieselbe Krankheit gemeint wird.

1529 entsteht „Eobanus Hessus Lectori“ („Eobanus Hessus an den Leser") - Widmungsgedicht von H. E. Hess zu Althamers Germaniakommentar. Andreas Althamer (1500 - ca. 1540), Huma- 
nist und Theologe, schrieb 1529 einen Kommentar zu Tacitus' „Germania“. Hess glaubt, beide Historiker haben ihre Qualitäten gleichermaßen offenbart, sowie im nützlichen Inhalt, als auch in stilistischer Sicht. Die Meinung ist wichtig in dem Sinne, dass die nationalen Leistungen in der Wortkunst hoch sind und es ist noch ein Schritt zur Behauptung der deutschen Poesie und zum Teil auch des deutschen Hexameters. Eben diesem Zweck dient auch in Georg Sabinus Ode „Apollo“ (1538) der Vergleich Melanchtons mit dem griechischen Gott der Künste.

Lateinische Hexameterverse finden wir in „Pasquillus vf den protestirenden Krig“ (1546), einem Beispiel der Makkaronika, wo zum Unterschied zu Brandt und zu einem erwähnten Anonym keine deutschen Verse den lateinischen, sondern lateinisierte Wörter mit deutschen Stämmen (ein Landgrafi, Reichstettites Narri, Gottswortum u. a.) den lateinischen Wendungen gegenübergestellt werden [DL 2001, 3: 155]. Makkaronisch ist auch „Benedictio Mensae in Pancketum“ („Tischsegen beim Bankett“, 1548) eines Unbekannten Verfassers, wo grobe familiäre Wörter lateinische Endungen bekommen (sauffite, fressendi) [ibid., 3: 158].

Ein interessantes Beispiel ist Konrad Gessners Übertragung des Gebets „Vater unser“ (1555) aus dem Lateinischen ins Deutsche mit Hilfe von deutschen Hexametern (vier Distichen, wo letzte zwei mit männlichen Klausuren ausgehen) und nebenbei auch von Endecasillabo. Uns interessiert der Hexameter:

O Vater unser der du dyn eewige wonung

Erhöchst inn himmeln dyn namen werde geheilget.

Zükumm vns dyn rich. Dyn will der thüe beschähen Vff erd als inn himmelen. <...> [ibid., 3: 173]

Der Aufklärer J. Ch. Gottsched (1700-1766) kritisierte Gessner nicht nur für seine Spondeen, die sich in der Tat als Jamben erweisen, sondern auch für seinen Gräzismus ,inn himmelen“, sowie dafür, dass er kurze Vokale für lange hält.

Gottsched findet den Hexameter sogar in Luthers Bibel (1534). Es ist auch nicht zufällig. Wie der erste leoninische Vers in Otfrieds Evangelienbuch „entdeckt wurde“, so sollte auch der große Luther den Hexameter als erster verwenden. Es kommt auch darauf an, dass beide Werke religiöse Texte sind, die den Hexameter den heidnischen Texten abgewinnen.

\section{- VV - - - - - - - VV-V}

Und Isa | ac scher | zet mit | seinem |Weibe Re | becca [Gottsched 1730: 471].

Johannes Claius von Hirzfeld (1535-1592) dichtet „Exemplum Carminis Heroici“ („Beispiel für ein Gedicht in Hexametern“, 1587), das gar keinen heroischen Inhalt hat, sondern scherzhaft ein Rätsel über den Turmhahn enthält.

Ein Vogel hoch schwebet / der nicht als andere lebet/

Nach keim Thier strebet / sich in allen Winden erhebet.

Vnd wenn die wüten / muss er denn fleissiger hüten / Wechst in fewers glüten / darff nicht als andere

brüten.

[DL 2001, 3: 236]

Schlussfolgernd sei es hervorzuheben, dass sich die Übergangsformen von den lateinischen zu den deutschen Hexametern im Humanismus durch die deutschen Themen und durch die gemischten lateinisch-deutschen Verse charakterisieren.

\section{Deutsche Hexameter und Distichen im 17. -} 18. Jahrhundert. Im 17. Jahrhundert, wie bekannt ist, herrscht der Alexandriner, der sogar in Sonetten verwendet wird. Die Dichter setzen fort lateinische Verse zu schreiben, darunter auch elegische Distichen. Der Theoretiker des Verses im 18. Jahrhundert J. Ch. Gottsched begann die Geschichte des Hexameters mit Luthers Bibel, erwähnte Gessner und bot seine Übersetzung von „Vater unser“ (1730) mit Hexametern an. Gottscheds Übersetzung ist viel flexibler als die von Gessner, weil sie nach fast 200 Jahren erschien.

Hör uns, Vater und Herr, der du den Himmel

bewohnest,

Daß dein Name bey uns über alles geheiliget werde,

Daß dein herrliches Reich bey uns auf Erden erscheine,

Und dein Wille von uns, eben so, als im Himmel geschehe.

Gib auch das tägliche Brodt, und vergib uns die sündlichen Schulden,

Wie wir auch unseres Theils den Schuldenern gerne vergeben.

Wende Versuchungen $a b$, und rett uns aus Gnaden, vom Uebel,

Denn dein ist das Reich, ja göttliche Macht und Herrlichkeit, Amen

[Gottsched 1730: 472]. 
In etlichen Paragraphen seines Buches („Versuch einer Critischen Dichtkunst“, 1730) urteilt Gottsched über den Hexameter im 17. Jahrhundert. Da das heroische Maß ohne Reim von deutschen Ohren als fremd empfunden wird, greifen einige Dichter zum Mischen von Hexameter und Pentameter, das heißt, sie schreiben lateinische Elegien und versuchen sie durch den Reim angenehmer zu machen. Zum Beispiel S. Birken, der Pegnizer Hirte, gab dafür ein Beispiel, das ihm nicht besonders gelang, so Gottsched [ibidem]. Deshalb führt ihn Gottsched in seinem Buch nicht an. Folgende Zeile aus einem Epigramm, die bei Birken ausgesucht werden kann, deutet tatsächlich darauf hin, als könne sie sowie als Alexandriner als auch als Hexameter gelesen werden.

Schau'mich an / der du lebst. Ich war ja / was du bist [DL 2001, 4: 207].

Die ersten deutschen Hexameter auf Grund des akzentuierten Prinzips mit dem Endreim begann man im Barock zu schreiben, aber die waren im 17. Jahrhundert einzeln und folgenlos. Nach Gottsched gibt Omeis ein gültiges Muster von Hexameter:

Was ein menschliches Herz, von innen und außen betrübet,

Werde durch Gottes Gewalt, künftig und itzo verjagt.

Was ihr redet und thut, das werde von beyden geliebet,

Bis der Tod zugleich beyden das Leben versagt [zit. nach Gottsched 1730: 473].

Aber Gottsched kritisiert die Versuche den Hexameter durch die Reime zu „verbessern“, und versucht seine These durch die Beispiele aus Psalmen, Gedichten von Tibul und Ovid, Milton und Opitz zu belegen. Ein passendes Muster findet er bei Opitz:

Rom und Athen war sonst ganz reich an Meistern und Künsten,

Doch was nützte die Zahl philosophischer Lehrer und Schüler,

Welche man irgend gesehn? O! was für ein thörichtes Wesen,

Was für ein albernes Zeug ward täglich in Tempeln getrieben?

Pallas erschrak, und Jupiter selbst, der Vater der Götter

Hatte nur Abscheu davor. Schwärmt, schwärmt nur, ihr rasenden Pfaffen! [ibid.: 475].
Im 18. Jahrhundert wurden nach wie vor unreine Hexameter geschrieben, nicht nur von $\mathrm{Uz}$ und Ewald von Kleist („Frühling“, 1749), sondern auch von anderen Dichtern, die Alexandriner mit Verwendung von Doppelsenkungen gebraucht haben, deswegen erschien der Hexameter mit Auftakt.

Der entscheidende Verdienst in der Einsetzung des Hexameters auf dem deutschen Boden gehört Klopstock („Messias“, 1748). Kayser stellt fest, dass der Hexameter seit „Messias“ zu einem repräsentativen epischen Vers wird (Kayser 1999: 24), obwohl er von Lenau, Ling, Spitteler dennoch ignoriert wurde. Man verwendete sogar in Homers Übersetzungen andere Metren. Der Eindruck Klopstocks Verse auf die junge Generation war überwältigend. In seiner „Dichtung und Wahrheit" sprach Goethe begeistert über „diese so natürlich ausgedrückten und doch so schön veredelten frommen Gefühle, diese gefällige Sprache“ [zit. nach: Breuer 1999: 195].

Durch den glänzenden Weg, der gegen die Erde sich kehret,

Floß, nach der Erden Erschaffung, vom himmlischen Urquell entspringend,

Ein verklärter ätherischer Strom nach Eden herunter.

Auf ihm, oder an seinem von Wolken erhobnen

Kam dazumal bald Engel bald Gott, Gestade, zum vertraulichen Umgang

Zu den Menschen [ibid.: 194].

Klopstock trat gegen die Regeln von Opitz und Gottsched und den alternierenden Wechsel von Hebungen und Senkungen im jambischen Vers und gegen den Verzicht auf den Wechsel von Länge und Kürze der antiken Verse auf. Er arbeitete seine Rezitationsmetrik heraus, die sich auf die Übereinstimmung des Wortsinns mit der Wortbewegung im Vers abzielte. Das versucht er im Epigramm „Der doppelte Mitausdruck“ zu erläutern und meint den Ausdruck der Wörter und den Mitausdruck der Versmöglichkeiten.

Leiserer, lauterer Mitausdruck der Gedanken des Liedes

Sei die Bewegung des Verses. So oft er diesem Gesetz nicht

Treu und hold ist, gehet er nur, um zu gehen; und verirrter

Tritt er einher, wenn er gar anwandert gegen den Inhalt <...> [DL 2001, 6:239]. 
Es fällt gleich auf, dass das Wort „Mitausdruck" zwei Hebungen (Haupt- und Nebenhebung) hat, dabei steht die Nebenhebung in der starken Position. "Nicht treu" wird durch die Grenze zwischen zwei Zeilen getrennt, das lässt ein Enjambement entstehen. Das Verb „gehen“ wird in einer Form verlängert und bekommt eine betonte und eine unbetonte Silbe - „gehet", aber in einer anderen Form wird es verkürzt, um den Akzent vor der Pause zu verstärken - „gehen“. Wir treffen hier den Reim, der den Schluss der vorangehenden und den Anfang der nachgehenden Zeile vereint: „verirrter" und "tritt er", der den „verirrten Reim" hervorhebt; es gibt auch ein alliterierendes Echo zwischen "gehen“ und „gegen“. Alle Fälle der unerwarteten Reimordnung und der Widerholungen unterstreichen den ironischen Sinn der Strophe.

Viele Verslehrer (Knörrich, Breuer, Arndt u. a.) analysieren eine Hexameterzeile von Klopstock, sich auf seine Idee der Einheit von Versfuß und Versgedanken sogar in dem kleinsten Kolon stützend.

Schrecklich erscholl der geflügelte Donnergesang in der Heerschar [zit. nach Breuer 1999: 193].

Breuer teilt die Zeile in vier Teile und findet für sie folgende metrische Analogien:

Schrecklich erscholl (-vv-) - Choriambus;

der geflügelte (vv-vv) - Pyrrhichius und Daktylus;

\section{Donnergesang (-vv-) - Choriambus;}

in der Heerschar (vv--) - steigender Jonikus.

In den Verslehren wird an diesem Beispiel aus Klopstock der steigernde und sinkende Jonikus veranschaulicht und die geringe Verbreitung dieses antiken Metrums betont, da es nicht den natürlichen Akzent im Deutschen anspricht [Knörrich 2005: 105]. In der Versverwirklichung lässt Klopstock verschiedene Freiheiten zu, wie Zeilen mit Pyrrhichien in der „Elegie“, die wir unterstrichen haben:

Nur mit einem verräthrischen Laut, nur mit einem der Töne <...>

Sag es mit einem durchdringenden Ach, das meinem Ach gleichet <...>

Dürft ich mein Auge zu dir einst, seeliger Himmel, erheben $<\ldots .>$

Göttliche, du liebest! Ach wenn du denn doch

auch kenntest... [DL 2001: 5, 223].

Nach Klopstock schreibt in Hexametern $\mathrm{zu}$ verschiedenen poetologischen Problemen
A. G. Kästner (1719-1800), der den Einschluss von lateinischen Buchstaben in den deutschen Text verhönt, weil in der Schweiz "y“ als „i“ gelesen wird:

Seht die epischen Zeilen, frei vom Maasse der Syllben, Frei vom Zwange des Reims, hart, wie Zyrchische

Verse,

Leer, wie Meissnische Reime; Seht, der glyckliche

Kynstler

Fyllt mit roemischen lettern, mit pythagorischen y y

Zum Ermyden des Lesers, besser zu nutzende

Bogen [DL 2001, 5: 267].

Aber der Hexameter stört nicht, sondern hilft G. Fr. Steudlin in „Die Gletscher bei Grindelwald“ erhabene Schönheit der Natur beschreiben (1785):

Ja! Ich hab euch geseh'n, die ihr auf Wirtembergs

Feste

Schon die staunende Seele zum Lobgesange

begeistert,

Ja, Ich hab euch geseh'n, Helvetiens Riesengebirge!

[DL 2001, 6: 196].

Einen neuen Anstoß zur Entwicklung des deutschen Hexameters im 18. Jahrhundert hat J. H. Voss (1751-1826) mit der Übersetzung Homers „Odyssee“ (1781) gemacht.

Sage mir, Muse, die Taten des vielgewanderten

Mannes,

Welcher so weit geirrt nach der heiligen Troja

Zerstörung,

Vieler Menschen Städte gesehn und Sitte gelernt hat

Und auf dem Meere so viel' unnennbare Leiden

erduldet,

Seine Seele zu retten und seiner Freunde

Zurückkunft [DL 2001, 6: 42].

Da Voss dem quantitaven prosodischen Prinzip folgte, wurden anstelle der Hebungen Digraphe, doppelte Vokale oder Vokale mit nasalen Konsonanten eingesetzt (viel (ge...), vieler Menschen Städte, gesehn, und). Die Übersetzung wurde polar wahrgenommen. Pro Voss äußerte sich der Romantiker A. W. Schlegel: „Voss ist unstreitig als der zweite Erfinder anzusehen, und sein Verdienst dabei ist unübersehlich groß" [zit. nach Moennighhoff 2004: 79]. Contra - der Klassiker J. W. Goethe: „Für lauter Prosodie ist ihm die Poesie ganz entschwunden" [ibidem]. Nach 25 Jahren, als Voss zum dritten Mal die Übersetzung überarbeitete, klang sie schon ganz anders - viel flexibler und poetischer. Ein Beispiel aus „Ilias“(1806):

Und siehe, der held Agamemnon 
Trieb maulthier'und männer daher aus den zelten des lagers, Holz vom walde zu führen; zugleich, ein edler

Eilte Meriones mit, des tapfern Idomeneus gebieter, kriegsfreund [Voss 1960: 231].

Hexameter bei den Klassikern, Romantikern und Postromantikern. Epigramm (gr.: Inschrift) wird seit dem 7. Jahrhundert v. Ch. in Hexametern oder Distichen aufgeschrieben. Das Epigramm geht in seiner Entstehung auf die Gelegenheitsdichtung zurück, wird aber im Laufe der Zeit zu einem literarischen Genre. Sein Ursprung wird durch seine barocken Namen angezeigt Xenien, Subscriptio, Epitaph, Glückwusch u. a.m.

Im Epigramm, das als Gratulation zur Hochzeit diente und von $\mathrm{K}$. W. Ramler "Ode an die Venus Urania“ (1770) genannt wurde, hört man die Intonation von Horaz' „Exegi monumentum“ dank zwei Akzenten auf dem letzten Wort:

Göttin Liebe! Die weiht heute dein Agathon,

Unsers Cyneas Sohn, seinen vollendeten

Tempel: Zeuch in dein Haus, Venus Urania,

Erstgeborne des Himmels, ein! [DL 2001, 6: 23].

In ein Buch der Stammgäste trägt J. K. Lavater folgendes Gedicht (,In ein Stammbuch") für ein junges Mädchen (insgesamt 11 Zeilen) ein, dessen Länge durch mehrere Daktylen bedingt ist:

Jünger der himmlischen Tugend! (Dein Blick voll Unschuld und Güte

Deine Stimme, dein Mund, und deine Rede

Daß ich dem Fühlenden sage) Jünger verspricht mir der himmlischen Tugend! [DL 2001, 6: 44].

Im folgenden Distichon aus „Xenien“ geht es um das Ganze, das uns an die Einheit von Hexameter mit Pentameter erinnert:

Immer strebe zum Ganzen, und kannst du selber kein Ganzes

Werden, als dienendes Glied schließ an ein Ganzes dich an [DL 2001, 6: 233].

Aus „Xenien“ stammt auch dieses belehrende poetologische Distichon, dem der gehobene Stil und Geist der Klassik eigen ist:

Jeden andern Meister erkennt man an dem, was er ausspricht,

Was er weise verschweigt, zeigt mir den Meister des Stils [ibid., 6: 234].

Goethe zieht größere Aussagen in Form eines Epigramms vor, wie z. B. „Sinngedichte“ (1791), wo er Venedig und seine Nachteile unterstreicht im Unterschied $\mathrm{zu}$ der üblichen Begeisterung in dem für deutsche Künstler typischen Diskurs über Venedig. Das Stadtbild stellt sich aus 12 Strophen von zwei bis acht Zeilen zusammen. Führen wir die kleinste, aber kaum weniger ironische an:

Hast du Bajä gesehn, so kennst du das Meer und die Fische,

Hier ist Venedig, du kennst nun auch den Pful und Frosch [ibid., 6: 241].

Als Idylle (gr.: eidillion - ein Bild, ein kleines Gedicht) wurde ein kleines Genregedicht bezeichnet, üblicherweise aus dem Landleben (Vergil, Horaz, Ovid). Es stammt aus der antiken Tradition der bukolischen Hirtendichtung (Ekloge).

Schiller unterschied zwischen der antiken Art von Vers und Idylle als der bestimmten Art der Darstellung der Sensibilität des sentimentalen Dichters. Aber zu seiner Lebenszeit kommen sie oft zusammen; wie bei Voss, Hölty, Sulzer u. a. Die Idylle „Das Landleben“ (1777) schreibt Hölty im asklepiadeischen Versmaß, als Epigraph wählt er ein Zitat aus Vergil („Flumina amem silvasque inglorius"):

Wunderseliger Mann, welcher der Stadt entfloh!

Jedes Säuseln des Baums, jedes Geräusch des Bachs, Jeder blinkende Kiesel

Predigt Tugend und Weisheit ihm! [ibid., 6: 94]

Beim Überblick des Goethe'schen Hexameters stellen wir fest, dass Hexameter auf verschiedene Weise angewendet wird. Im Lehrgedicht «Reinecke Fuchs» (1793) folgt Goethe noch Klopstocks Muster, während er miteinander verbundene sprachliche Einheiten und gleichzeitig Verseinheiten mit paralleler Wiederholung (1. - 2. Zeilen) gebraucht.

Pfingsten, das liebliche Fest, war gekommen: es grünten und blühten

Feld und Wald; auf Hügeln und Höhn, in Büschen und Hecken

Übten ein fröhliches Lied die neuermunterten

Vögel [ibid., 7: 13].

Anders bedient er sich des Hexameters in der bukolischen Dichtung «Hermann und Dorothea» (1797). Er schreibt mit den flexibleren Hexametern, die Mannigfaltigkeit von Zäsuren aufweisen. Um die Monotonie des Verses zu beseitigen, zerlegt Goethe die Zeile in 2 Sätze (2. Zeile), wobei er weiter Enjambements, Inversionen, Ein- 
führungssätze u. a. m. verwendet. Zu der Emotionalität tragen auch Ausrufesätze bei:

Hab ich den Markt und die Straßen doch nie so einsam gesehen!

Ist doch die Stadt wie gekehrt! Wie ausgestorben! Nicht fünfzig,

Deucht mir, blieben zurück von allen unsern Bewohnern.

Was die Neugier nicht tut! So rennt und läuft nun ein jeder,

Um den traurigen Zug der armen Vertriebnen zu sehen [ibid., 7: 25].

Nach einer Version wurde Goethe durch Voss' Übersetzungen, nach einer anderen durch Winkelmanns Werk („Geschichte der Kunst des Altertums“, 1764) und durch die Reaktion auf den Streit um Homer und F. A. Wolffs Kritik in „Prolegomena ad Homerum" (1795) inspiriert bei der Schöpfung eines unbeendeten Poems «Achilleis», das mit Hectors Tod beginnt:

Hoch zu Flammen entbrannte die mächtige Lohe noch einmal,

Strebend gegen den Himmel, und Ilios Mauern erschienen

Rot durch die finstere Nacht; der aufgeschichteten Waldung

Ungeheures Gerüst, zusammenstürzend, erregte

Mächtige Glut zuletzt. Da senkten sich Hectors Gebeine

Nieder, und Asche lag der edelste Troer am Boden

[ibid.: 7, 60].

Weiter hatte Goethe vor, über die Liebe Achilles zu Polyxene zu schreiben und das Poem mit Achilles'Tod zu beenden, aber ließ seinen Plan unerfüllt. Was war der Grund dazu, bleibt unbekannt, aber vermutlich passte die Gestalt des liebenden Achilles mehr zu dem Drama als zum epischen Vers.

Neben Epigrammen und Idyllen haben die Klassiker auch Elegien geschriebn. Ein Beispiel dafür - die erste von Goethes „Römischen Elegien“ (1788-90), die auf antike erotische Elegien zurückgehen. In der folgenden Elegie denkt der Dichter über die römische Architektur nach:

Saget Steine mir an, o! sprecht, ihr hohen Paläste!

Straßen redet ein Wort! Genius regst du dich nicht? Ja es ist alles beseelt in deinen heiligen Mauern,

Ewige Roma; nur mir schweiget noch alles so still [ibid., 6:286].

Schiller hat seine „Nänie“ (lat.: Grablied) (1799) dem Helden Achilles gewidmet und bezog sich dabei auf die Vorstellung der alten Griechen von Schönheit und Mut.

Auch das Schöne muss sterben! Das Menschen und Götter bezwinget,

nicht die eherne Brust rührt es des stygischen Zeus [ibid., 6:306].

In einer anderen Elegie handelt es sich bei Schiller um „Die Dichter der alten und neuen Welt" (1795):

Sagt, wo sind die Vortrefflichen hin, wo find'ich die Sänger,

Die mit dem lebenden Wort horchende Völker entzückt,

Die vom Himmel den Gott, zum Himmel den Menschen gesungen

Und getragen den Geist hoch auf den Flügeln des Lieds? [ibid., 6: 291].

In der "Geschichte der deutschen Elegie“ kommt Beissner zur Schlussfolgerung, dass „Wechsel zwischen der hellen Melodie hexametrischen Vorwärtsdrängens und dem dumpf verhaltenden Doppelstoß des Pentameters das elegische Empfinden vertieft" [Beissner 1991: 76]). Diesen Wechsel veranschaulicht Schiller in seinem elegischen Vers:

Im Hexameter steigt des Springquells flüssige Säule, Im Pentameter drauf fällt sie melodisch herab

[DL 2001, 6:334].

Bei Claudius hat es im Vergleich zu Schiller mehr ironisch geklungen:

Im Hexameter zieht der ästhetische Dudelsack Wind ein;

Im Pentameter drauf läßt er ihn wieder heraus [ibid., 6: 214].

Schiller war ein großer Meister des Distichons, er begann seit 1795 dieses Metrum zu pflegen, ein halbes Jahr vor der Bekanntschaft mit Goethe und auch später hat er auf das Versmaß nicht verzichtet, was seine philosophische Lyrik beweist („Elegie“, mehr als „Spaziergang“ bekannt):

Sey mir gegrüßt, mein Berg mit dem röthlich strahlenden Gipfel! [ibid., 6:290].

Mit Distichen hat Schiller über Metren und feste Strophen geschrieben, über die Stanze hat er auch folgendes Distichon gedichtet:

Stanze, dich schuf die Liebe. Die zärtlich schmachtende. Dreymal

Fließt du schamhaft und kehrst dreymal verlangend zurück [ibid., 6: 295]. 
Über den Hexameter hat er auch ein Distichon geschaffen:

Schwindelnd trägt er dich fort auf rastlos strömenden Wogen,

Hinter dir siehst du, du siehst vor dir nur Himmel und Meer. [ibid.: 6, 296].

Nach Kayser hat Schiller die reinsten Hexameter in der deutschen Dichtung geschrieben [Kayser 2005: 104]. Außerdem benutzt Schiller die antithetischen Möglichkeiten des Pentameters und ihre Widerspiegelungen (Hinter dir siehst $d u, d u$ siehst vor dir). Brüder Schlegel wirkten in Schillers Zeitschrift „Die Horen“ und Auseinandersetzungen mit den Romantikern hat Schiller auch in Form des Distichons geäußert:

Lange neckt ihr uns schon, doch immer heimlich und tückisch,

Krieg verlangtet ihr ja, führt ihn nun offen, den Krieg. [ibid.: 7, 297]

Die Auseinandersetzung zwischen einem Klassiker und einem Romantiker veranschaulicht Kayser an Hölderlins Gedicht „Brot und Wein“, von dem in jener Zeit nur eine Strophe unter dem Titel „Die Nacht" bekannt war. Brentano war von dieser Strophe bezaubert:

...die Nacht kommt,

Voll mit Sternen und wohl wenig bekümmert um uns,

Glänzt die Erstaunende dort, die Fremdlingin unter den Menschen

Über Gebirgeshöhn traurig und prächtig herauf [zit. nach Kayser 1991: 126].

Diese Zeilen zitierte Brentano in seinen Briefen und schloss mit einer leichten Variation als ein Prosastück in sein Märchen ein „Gockel, Hinkel und Gakeleia“. Für den Anfang benutzt er statt des Präsens das Präteritum (die Veränderungen werden fett hervorgehoben - T. A.):

Die Nacht kam, trunken von Sternen und wohl wenig bekümmert um uns

glänzte die Erstaunende dort, die Fremdlingin unter den Menschen,

über Gebirgshöhn traurig und prächtig herauf? [ibidem].

Dank dem Präteritum erscheinen plötzlich drei nicht betonte Füße (Tribrachia), die in einem Hexameter oder Pentameter unmöglich sind und die sie einfach in die Luft jagen. Und in der letzten Zeile, aus einem 6-Fuss-Vers bildete sich plötzlich ein 5-Fuss-Vers, die Zäsur verschwand, der Pentameter brach zusammen und damit das
Distichon. Kayser kommt zu dem Schluss, dass Brentano so in "Des Knaben Wunderhorn“ auf "fließenden Rhythmus" umschaltet, was zur Zerstörung der Strophe führt - und diese hat „eine Auflösung der Strophe zu Folge" [ibid.: 127], aber es war eine große Leistung der romantischen Dichtung im Vergleich zu den mehr statischen klassischen Formen.

Hölderlin benutzt den Hexameter in „Archipelagus", in dem er die Zeile in 2-3 Teile gliedert, was dem Vers Beweglichkeit und der Intonation Aufregung verleiht:

Aber weh! es wandelt in Nacht, es wohnt, wie im Orkus,

Ohne Göttliches unser Geschlecht. Ans eigene Treiben

Sind sie geschmiedet allein, und sich in der tosenden Werkstatt

Höret jeglicher nur und viel arbeiten die Wilden

Mit gewaltigem Arm, rastlos, doch immer und immer

Unfruchtbar, wie die Furien, bleibt die Mühe der Armen [DL 2001: 7, 92].

Hölderlin benutzt das elegische Distichon im Klagelied wie „Menons Klagen um Diotima“:

Täglich geh'ich heraus, und such’ ein Anderes immer,

Habe längst sie befragt alle die Pfade des Lands;

Droben die kühlenden Höhn, die Schatten alle besuch' ich,

Und die Quellen; hinauf irret der Geist und hinab, Ruh' erbittend; so flieht das getroffene Wild in die Wälder,

Wo es um Mittag sonst sicher im Dunkel geruht; Aber nimmer erquickt sein grünes Lager das Herz ihm,

Jammernd und schlummerlos treibt es der Stachel umher [DL 2001: 7, 31]. „Distichen“ (1798) begegnet man bei Novalis, obwohl manche Verslehrer über die Gleichgültigkeit der Romantiker dem Hexameter gegenüber sprechen [Kayser 1991; Knörrich 2005]. Das vierte Distichon ist als Epigramm verfasst:

Einem gelang es - er hob den Schleier der Göttin $\mathrm{zu}$ Sais -

Aber was sah er? Er sah - Wunder des Wunders sich selbst [Novalis 1985: 52].

Als Distichen hat Novalis auch „Erkenne dich selbst“, „Letzte Liebe“, „An die Schwester Karoline im Namen der Mutter" u. a. gestaltet. Die Kürze der Form weist auf Epigramm als Genrequel- 
le hin. Kleists zweizeilige „Epigramme“ aus den Journalen „Phöbus“ и „Berliner Abendblätter“ zeigen auch der Thematik nach sein organisches Verhältnis zur Antike und Klassik. Fast alle sind sie im Vergleich zu Goethes Epigrammen betitelt - „Der Ödip des Sophokles“, „Der Areopagus“, „Demosthenes, an die griechischen Republiken" u. a. m. Darunter gibst es auch solche, die sein eigenes Schaffen angehen und akute Probleme seiner Zeit und Literatur zur Sprache bringen. Das Epigramm „Die Marquise von O..." ist aus der Perspektive eines Philisters geschrieben:

Dieser Roman ist nicht für dich, meine Tochter. In Ohnmacht!

Schamlose Posse! Sie hielt, weiß ich, die Augen bloß zu [Kleist 1983: 4].

Die Postromantiker Fr. Rückert und A. Platen als Nachfolger der Klassiker und Romantiker versuchten verschiedene östliche und antike $\mathrm{Me}$ tren und Genres zu pflegen. Rückert scherzt im Genre des Epigramms über die "Grammatische Deutschheit" (1819):

Neulich deutschten auf deutsch vier deutsche Deutschlinge deutschend,

Sich überdeutschend am Deutsch, welcher der

Deutscheste sey [DL 2001, 7: 245].

Rückert erinnert seine Leser auch an den leoninischen Vers:

Über die Gipfel der Berge zu streben, / das wär'ein Leben! [ibid., 7: 246].

Teilweise im parodistischen Sinne gebraucht den Hexameter Mörike. Ein Zitat aus der dramatischen Szene unter dem Namen „Häusliche Szene“ mit den Autorenanweisungen („Schlafzimmer. Präzeptor Ziborius und seine junge Frau. Das Licht ist erlöscht" [Mörike 1965: 302]) führen wir an:

„Schläfst du schon, Rike?“ - „Noch nicht“. -

„Sag, hast du denn heut' die Kukumern

Eingemacht?“ - „Ja“. - „Und wie viel nahmst du mir Essig dazu?" -

„Nicht zwei völlige Maß“. - „Wie? Fast zwei Maß?

Und von welchem

Krug? Von dem kleinen doch nicht, links vor dem Fenster am Hof “. -

„Freilich“. - „Verwünscht! So darf ich die Probe nun noch einmal machen,

Eben indem ich gehofft, schon das Ergebnis zu sehn" [Ibidem].

Am Ende dieser Szene kommen die Ehegatten auf den Gedanken, auf einmal miteinander über
Hexameter und Pentameter zu sprechen, dabei spricht der Gatte mit Hexametern und die Gattin - mit Pentametern. In der Autorenanweisung sagt Mörike ironisch: „Pause. Der Mann wird unruhig; es peinigt ihn offenbar, das Distichon nicht geschlossen zu hören oder es nicht selber schließen zu dürfen. Nach einiger Zeit kommt ihm die Frau mit Lachen zu Hilfe und sagt"<...> [ibidem]. Die Ehegattin kommt dem Gatten zu Hilfe und schließt das Distichon mit dem Pentameter, was den Mann freundlich einstimmt und das Gespräch mit Lächeln und Kuss abschließt.

Mörike hat verschiedene Genres gepflegt Epigramme, Idyllen, Elegien: „Göttlicher Wink“, „Im Park“, „Nachts am Schreibpult“. Es gibt bei ihm eine Reihe von Idyllen: „Im Weinberg“, „Der Rheinfall“, „Märchen vom sichern Mann“, „Die schöne Buche“. Bei Mörike gibt es auch eine größere Idyllenform - „Idylle vom Bodensee oder Martin Fischer. In sieben Gesängen" mit dem epischen Anfang:

Dicht am Gestade des Sees im Kleefeld |steht ein verlaßnes

Kirchlein unter den Höhn, die, mit Obst und Reben bewachsen,

Halb das benachbarte Kloster |und völlig das Dörfchen verstecken,

Jenes gewerbsame, das weitfahrende Schiffe beherbergt [Mörike 1965: 7].

A. von Platen setzt das Thema des Fischerlebens in seiner Ekloge „Das Fischermädchen in Burano“ (1833) fort, wo er das ihren Mann vom Meer erwartende Mädchen in der Gesellschaft von ihren Freundinnen darstellt und das folgende Distichon als Anfangs- und Schlusszeile gebraucht:

Strickt mir fleißig am Netz, ihr Schwestern! Es soll's der Geliebte

Heut noch haben, sobald im besegelten Nachen er heimkehrt [Platen 1982: 545].

Viele Epigrammarten zu verschiedener Thematik kann man noch bei Mörike finden: das sind Epitaphen („Auf das Grab von Schillers Mutter"), Aufschriften zu den Porträts („Johann Kepler“, „Maschinka“, „Das Bildnis der Geliebten“), Streite ("Muse und Dichter"), Gelegenheitsgedichte zum Geburtstag („An meine Mutter"). Aber mit Mörike endet die Geschichte der lyrischen Idylle, für die ein schönes Muster „Wald-Idylle“ sei:

Unter die Eiche gestreckt, im jung belaubten Gehölze

Lag ich, ein Büchlein vor mir, das mir das 
lieblichste bleibt. Alle die Märchen erzählts, von der Gänsemagd und vom Machandel Baum und des Fischers Frau, wahrlich, man wird sie nicht satt [ibid.: 103].

Hexameter im 20. Jahrhundert. Der Hexameter ist vor allem das poetische Versmaß des Epos (heroischer Hexameter). In anderen Fällen erscheint der Hexameter hauptsächlich in Gedichten von großem Format, zum Beispiel im Lehrgedicht, aber sie enthalten ständig satirische Anfälle (wir werden es am Beispiel von Bobrowski sehen). Um die Wende der Epochen versuchte G. Hauptmann den Hexameter in "Till Eulenspiegel“ (1927/8) wiederzubeleben.

Nur herein, nur hereinspaziert! meine Herren und Damen!

ohne Furcht, ohne Zagen! Der Krieg - Gott sei Dank - ist vorüber!

Gold ist freilich nicht im Lande: das haben die Schweizer,

vor allen die Wall Street. Wir aber, wir haben das Nachsehn! - [Hauptmann 1928: 18].

In der Moderne ist der Hexameter äußerst selten, er tritt bei Dichtern auf, die mit verschiedenen Metren arbeiteten, wie die österreichischen Dichter Saar, Weinheber, Bachmann. Ein Zyklus aus 15 Elegien unter dem Namen „Wiener Elegien“ von F. L. A. Saar (1833-1906) ist den Erinnerungen an das Wien seiner Kindheit, mit Feiertagen, Dichtern und Schriftstellern, reich und arm, mit Parks und Schlössern und der Wiener Moderne gewidmet. Folgenderweise äußert sich der Autor über den Unterricht in Latein und Griechisch und über das Verständnis des Hexameters:

Und nun ging es, o Qual! an lateinische, griechische Pensa,

Bebenden Fingers gezählt ward des

Hexameters Maß.

Marternde Sorgen des Schülers, die Angst vor der schlechteren Note -

Jetzt noch fühl' ich sie nach, schreit' ich hier sinnend vorbei! [Saar].

Es war auch notwendig, an diesen Zyklus des österreichischen Schriftstellers und Dichters im Zusammenhang mit der Ansicht zu erinnern, der Hexameter komme in der Moderne selten vor [Breuer 1999; Knörrich 2005; Moennighoff 2004]. Und hier wäre die Meinung von Nietzsche, einem
Philosophen und Kenner der Antike, einem Dichter, der verächtlich alternierende Verse „ticktack“ und die betonte Silbe „Hopsassa Iktus“ nannte, nicht ohne Interesse. In einem Brief an Carl Fuchs im August 1888 erklärt er den Unterschied zwischen dem Rhythmus bei den Alten und den Deutschen. Nietzsche schreibt: „Unser Rhythmus ist ein Ausdrucksmittel des Affekts: der antike Rhythmus, der Zeitrhythmus, hat umgekehrt die Aufgabe, den Affekt zu beherrschen und bis zu einem bestimmten Grade zu eliminieren. <...> In summa: unsere Art Rhythmik gehört in die Pathologie (Lehre von der Erregung der Leidenschaften), die antike zum Ethos (Besänftigung der Leidenschaften)“ [zit. nach Breuer 1999: 234].

Trotzdem begegnet man den Hexametern auch später, in der Zeit zwischen zwei Kriegen, zum Beispiel bei J. Weinheber, einem Meister der Versform. Sein Gedicht („Auf das Unabwendbare“) aus dem Buch („Anfechtung und Trost im deutschen Gedicht“, 1938) ist in Distichen abgefasst.

Der hier weiß seinen Weg und geht ihn mit Stolz. Oder tückisch

Schiebt der sein Planvolles vor. Oder ihn treibt nur die Nacht.

Aber blind sind wir alle. Sehn nicht, wie uns das Höhere

Ringelbahn führt und es kommt jeder im Kreise vorbei

An dem starrenden Antlitz, und jeder noch einmal und wieder,

Und mit jedem Mal reift größer, gewisser die Angst.

Zwings, versuchs doch! Heb den tappigen Fuß aus dem Bügel

Deines hölzernen Pferds: Spring und verlass dich auf Gott! [Weinheber 1983: 273].

Gertrud Kolmar schließt einzelne hexametrische Zeilen in die Gedichte („Der Sohn“ u. a.). Ganz hexametrisch und mit vierzeiligen Strophen werden „Die Hässliche“ und „Der Schwimmer"verfasst, das Letzte vermutlich als Allusion an Schillers Ballade „Der Taucher“:

Aus der umplätscherten Brücke, jenseits behütender Schranke,

Ganz am Ende, gereckt, die Arme aufwärts gebreitet,

Ernst am weißlichen Himmel, erschauernder Vogelgedanke,

Standest du, schon zum Flug, zum Wurf in Leere 
gespreitet [Kolmar 1980: 254].

In der Nachkriegszeit schrieb H. E. Holthusen seine "Trilogie des Krieges“ aus der Gedichtsammlung „Hier in der Zeit“ (1949) in Hexametern. Die Elegie (127 Zeilen), die aus drei Teilen besteht, in denen der Drang nach Osten, die Umkreisung der deutschen Armee bei Stalingrad und die Heimkehr nach dem Niederschlag dargestellt werden, kann als Versuch gelten, sich des Kriegs zu besinnen. Die Schuld der Deutschen vor der Welt und die Befreiung von der faschistischen Barbarei ist das Hauptthema der Elegie.

Trennte der Mensch, ein Feind seiner selbst, die Zeit in zwei Teile,

Trennte in Frieden und Krieg sein heillos verwickeltes Dasein.

Ach, es war die Stunde der Schlachtung am düsteren Stein der Geschichte,

Während Vernunft, sich verschleiernd, zurücktrat.

Die Völker sind schrecklich [Holhusen 1989: 11].

R. A. Schröder und B. Brecht verkörpern didaktische und belehrend-ironische Tendenz im deutschen Hexametervers. Schröder als Kenner der antiken Literatur und Übersetzer der "Ilias" und „Odyssee“ Homers, der Werke von Vergil und Horaz, beherrschte den Hexameter und schrieb in diesem Versmass. Kayser hält Weinheber und Schröder für die „bedeutendsten Dichter in den antiken Odenmaßen des 20. Jahrhunderts" [Kayser 1991: 50] und beweist es mit dem Zitat aus der Horazischen Ode im asklepiadeischen Versmaß von Schröder: Sommer, Sommer! Dieweil läutert im Mittag schon Herbst des scheidenden Jahrs tränenbeträuft

Geschenk,

Bricht denn, ehe das Dunkel

Kam, die berstende Frucht vom Baum [ibidem].

Und Kayser hat recht, wenn er glaubt, diese „Verse folgen nun dem natürlichen Gefühl der deutschen Sprache“ [ibidem], aber auch der Verfallsstimmung der Nachkriegszeit.

Einen ganz anderen Gebrauch findet für seine Hexameter Brecht. Nach dem Muster von Lukrez',De rerum natura“ begann er Anfang 1945 am Lehrgedicht „Das kommunistische Manifest“ $\mathrm{zu}$ arbeiten, das heißt, an der Umgestaltung von Marx'Manifest in Hexametern. Die vermutlichen Titel hießen: „Von der Natur des Menschen“ oder „Von der Unnatur der bürgerlichen Verhältnisse“. Ende des Krieges und der 100. Erscheinungstag des Manifestes waren Anstöße zu dieser Idee. 1953 erkannte Brecht in „Memorandum“, dass
Virgil',Georgica“ und Lukrez',De rerum natura" in Übertragung von J. H. Voss und K. L. von Knebel den Deutschen zeigten, wie die Weltanschauung im Lehrgedicht geäußert werden kann. „Der Hexameter ist ein Versmaß, das die deutsche Sprache zu den fruchtbarsten Anstrengungen zwingt. Sie erscheint deutlich "gehandhabt", was das Lernen sehr erleichtert. <...> der große Kunstverstand der Alten entwickelt sich an großen Inhalten" [zit. nach Suvin 2008: 610].

Deautomatisierung oder die Verfremdung des prosaischen Textes in Hexametern konnte die Wahrnehmung des vergessenen Textes erfrischen. Außerdem könnte Marx'Text durch die neuen Begriffe der Theorie der zyklischen Krisen, die zu den Kriegen führen, aktualisiert werden. Der neue Text wurde durch Wiederholungen, Parallelismen, Personifikationen, rhetorische Fragen statt der rationellen Struktur der wissenschaftlichen Thesen dramatisiert. „Das wird durch mannigfaltige Mittel erreicht: durch syntaktischen Parallelismus an Stelle der marxschen logischen Subordination, durch weitergehende Verwendung der Personifizierung und der dynamischen Handlung <...> " [ibid.: 612].

Kriege zertrümmern die Welt und umgeht zwischen den Trümmern

Sichtbar und groß ein Gespenst, und nicht erst der Krieg hat's geboren.

Auch im Frieden schon ward es gesichtet, den Herrschenden schrecklich Aber freundlich den Kindern der Vorstadt. In ärmlicher Küche

Lugte es oft, kopfschüttelnd, voll Zorn, in halbleere Töpfe.

Oft die Erschöpften passte es ab vor Gruben und Werften.

Freunde besucht es im Kerker, passierend ohne Passierschein

Oftmals. Selbst in Kontoren wird es gesehen, und im Hörsaal

Wird es gehört. Zu Zeiten dann stülpt es von Stahl einen Hut auf

Steigt in riesige Tanks und fliegt mit tödlichen Bombern.

Vielerlei Sprachen spricht es, alle. Und schweiget in vielen.

Ehrengast in den Hütten sitzt es, Sorge der Villen Alles zu ändern und ewig zu bleiben gekommen; sein Name

ist Kommunismus. <...> [ibid.: 614] 
Im Vergleich zu dem 19. Jahrhundert hat sich der Diskurs verändert (Tanks, Bomber), ein Teil davon ist auch erhalten (Gruben und Werften, Kontoren und Kerker), darunter antithetische Schlüsselwörter aus dem Text des Originals: Gespenst, Hütten - Villen, Krieg - Frieden, Herrschende - Erschöpfte. Zweifel von den Arbeitern verstanden zu werden, bewog Brecht im September 1945 auf diese Arbeit zu verzichten, obwohl der Dichter noch oft dazu zurückkehrte und sie umarbeitete.

das Proletariat muss /

Unterste Schicht der Gesellschaft, um sich zu erheben, den ganzen /

Bau der Gesellschaft zertrümmern mit all seinen oberen Schichten. /

Abschütteln kann sie die eigene Knechtschaft nur abschüttelnd alle /

Knechtschaft von allen. [ibid.: 615]

Der Dramatiker aus der ehemaligen DDR, Brechts Schüler Heiner Müller (1929-1995), hatte Goethe und Schiller als seine Orientiere nicht nur in der Dramatik, sondern auch in der Dichtung gehabt. Davon zeugt sein zweiteiliges Lehrgedicht «Geschichten von Homer»:

Häufig redeten und ausgiebig mit dem Homer die

Schüler, deutend sein Werk, ihn fragend

um richtige Deutung.

Denn es liebte der Alte immer sich neu zu entdecken

Und gepriesen geizte nicht mit Wein und

Gebratnem.

Kam die Rede, beim Gastmahl, Fleisch und Wein, auf Thersites

Den Geschmähten, den Schwätzer, der aufstand in der Versammlung

Nutzte klug der Großen Streit um das größere

Beutstück Sprach:

Sehet an den Völkerhirten, der seine Schafe

Schert und hinmacht wie immer ein Hirt, und zeigte die blutigen

Leeren Händ der Söldner als leer und blutig den Söldnern [Müller].

Weder Achilles noch Thersites wird zum Helden seines Gedichtes, sondern Homer, in dem wir Brecht erkennen können. Die Situation eines Schmauses mit den Schülern, das Gleichnis über den Bogen, das Genre des Lehrgedichtes, das Problem der Wahrheit - alles erinnert den Leser an Brecht.

Die ironische Intonation verwendet ein anderer DDR-Dichter - J. Bobrowski in einem bö- sen Epigramm, wo er die Leute verhöhnt, die den Krokodils-Tränen glauben:

Oh ungeheure Erschütt'rung! Oh seht nur, die Krokodile

weinen, Träne um Trän'rollt zu den Wangen herab. Aber das eben zerfleischte, hinuntergeschlungene Opfer?

Seht diesen Krokodils-Jammer, und fragt nicht, weint mit! [DL 2001, 10: 40].

Bei der österreichischen Dichterin I. Bachmann finden wir elegische Hexameterderivate mit Auftakt oder Trimeter mit doppelten Senkungen im Gedicht «Eine Art Verlust».

Gemeinsam benutzt: Jahreszeiten, Bücher und eine Musik.

Die Schlüssel, die Teeschalen, den Brotkorb, Leintücher und ein Bett.

Eine Aussteuer von Worten, von Gesten, mitgebracht, verwendet, verbraucht.

Eine Hausordnung beachtet. Gesagt. Getan.

Und immer die Hand gereicht [DL 2001, 10: 38].

In der Dichtung der ehemaligen DDR hat nicht nur J. R. Becher, sondern auch Hans Cibulka Elegien geschrieben, wie im Gedicht "Deutschland 1945“:

Schon seit einigen Tagen durchstreichen die Krähen den Garten, aus dem nackten Geäst wächst der Gedanke, der Wind.

Langsam neigt sich der Erde silberne Tröstung hernieder,

beugt den Nacken der Zeit in ihr eisiges Joch.

[DL 2001: 10, 78].

Knörrich meint, „die spezifische Eigenart des Pentameters geht verloren, wenn er im Distichon, entsprechend seinem Namen, durch einen fünffüßigen Daktylus ersetzt wird" [Knörrich 2005: 167], wie zum Beispiel in H. Kahlaus „Elegie“ (in dem Gedichtband "Mikroskop und Leier“, 1964).

Unfreundlich wird mir mitunter das eigene Heim für die Dichtung,

weil es nicht Raum hat für zärtliche sorglose Träume [ibidem].

Knörrich hat nur teilweise recht. Wenn die Pause nach "für" gemacht wird, wird der Pentameter wiederhergestellt, obwohl die nötige Katalektik dennoch fehlt.

Wenn wir über die modernen Elegieformen nachdenken, muss man Arndts Meinung über die Doppeldeutigkeit des Wortes „Elegie“ zustim- 
men: einerseits sei es ein Gedicht, das aus elegischen Zeilen besteht. Das heißt, dass in erster Linie nicht die Thematik, sondern die Form beachtet wird. Andererseits sei es ein Gedicht mit einer bestimmten Stimmung - klagevoll, traurig, wehmütig [Arndt 1984: 192]. Es wird aber mit einem anderen, nicht elegischen Maß gedichtet, wie Goethes "Marienbader Elegie“ oder Rilkes „Duineser Elegien“. In Brechts „Buckower Elegien“ (1953) aber führt manches Gedicht, wie «Der Rauch», auf die Distichen zurück, abgesehen von ihrer transformierten Form:

Das kleine Haus unter Bäumen am See / Vom Dach steigt Rauch/

Fehlte er/ Wie trostlos dann wären /

Haus, Bäume und See [DL 2001, 10: 49].

Wenn wir unter den gegenwärtigen Dichtern einen Hexameteranhänger suchen, gehört Durs Grünbein zu den raren Autoren, die sich für alte und verschiedenartige Metren interessiert ( $\mathrm{z}$. B. in seinen Büchern „Den teuren Toten“, 1994, „Nach den Satiren“, 1999). In seinem transformierten Sonett "Memorandum“ (mit Allusion an Brechts „Memorandum") sind die erste und die letzte Zeile mit Hexametern geschrieben.

Alles geht weiter, |nicht erst seit heute, vor allem der Krieg, <...>

Glaubte das Ende der Dichtung nah, nicht zum letzten Mal [Grünbein 2003: 324].

Alban Nikolai Herbst (Pseudonym für Alexander Michael von Ribbentrop, geb. 1955), ein gegenwärtiger Schriftsteller, Übersetzer, Dichter, Blogger, benutzt in seiner Dichtung antike Versformen. Im dritten Band („Argo. Anderswelt“) seiner Romantrilogie „Anderswelt" lässt er seinen Helden unter dem Namen Achilles, oder gekürzt Hill Borkenbrod, handeln, und teilweise in dem hexametrischen Vers erzählt er seine Geschichte:

„So wuchs des Peleus" Sohn auf, der Thetis opalnem Gelege meeresgezeugter Schlupf Achill, der links immer lahmte, fersenwund seit dem Bootsbruch; ein Splitterchen steckte noch lange drin, das er selbst sich herausschnitt, blind fast, taub fast vor Schmerzen,

denen aber ein andrer Schmerz, der ihm schuppend im Rücken, juckend festsaß, entgegenstand und von dem er nicht wußte, was er war, noch was er war, selbst, der zu künden geschickte

Barde, voll der achäischen Lieder, um Vater zu werden,

Niams Vater, der rächenden Lamia, euch zu befreien.

Hätt ein Gott sie gezeugt, wer sicherte Göttern den Äther? [DL 2001, 10: 89].

Schlussfolgerung. Nachdem die Entwicklungsgeschichte des Hexameters auf deutschem Boden nachvollzogen worden ist, kann man folgende Stationen ausgliedern. Erstens beginnt die Geschichte mit dem Gebrauch des leoninischen Hexameters (auch als leoninischen Pentameter oder Vers genannt). Zweitens wird die Geschichte intensiv von Humanisten in Form eines gemischten latein-deutschen Hexameters entwickelt. Im Barock kommt der Hexameter einzeln vor, weil in der Dichtung fast überall der Alexandriner vorherrscht. Im 18. und in der ersten Hälfte des 19. Jahrhunderts werden mehrere Gedichte in Hexametern verfasst, die sich der deutschen Versmetrik anpassen, und solche Genres wie Epigramm, Elegie und Idylle werden in Form verschiedenartiger Subgenres entwickelt. Im 20. Jahrhundert wird auf Hexameter nicht völlig verzichtet. Die erwähnten Genres werden ironisch behandelt und transformiert. Der Hexameter selbst wird gelockert, aber dennoch mit Referenz auf die reiche Tradition in der deutschen Hexameterdichtung gebraucht.

\section{Literatur}

Гаспаров, М. Л. Метр и смысл. Об одном из механизмов культурной памяти / М. Л. Гаспаров. - М. : РГГУ, 1999. $-289 \mathrm{C}$.

Гаспаров, М. Л. Очерк истории европейского стиха / М. Л. Гаспаров. - М. : Наука, 1989. - 303 с.

Томашевский, Б. В. Теория литературы. Поэтика / Б. В. Томашевский. - М. : Аспект Пресс, 2001. - 334 с.

Холшевников, В. Е. Основы стиховедения. Русское стихосложение / В. Е. Холшевников. - СПб. ; М. : Академия, 2002. - 208 c.

Althochdeutsches Lesebuch (AL) / Hrsg. von Braune-Ebbinghaus. - Tübingen : Niemeyer, 1965. - 187 S.

Arndt, E. Deutsche Verslehre: Ein Abriss / E. Arndt. - 9. Aufl. - Berlin : Volk und Wissen, 1984. - 229 S.

Bantel, O. Grundbegriffe der Literatur / O. Bantel. - 4. Aufl. - Frankfurt a. M. : Hirschgraben, 1965. - 103 S. 
Behrmann, A. Einführung in den neueren deutschen Vers von Luther bis zur Gegenwart / A. Behrmann. - Stuttgart : Metzler, 1989. - 179 S.

Beissner, Fr. Geschichte der deutschen Elegie / Fr. Beissner. - Berlin : Gruyter, 1991. - 240 S.

Breuer, D. Deutsche Metrik und Versgeschichte / D. Breuer. - 4. Aufl. - München : Fink, 1999. - 414 S.

Bunge, H. Das Manifest von Bertolt Brecht / H.-J. Bunge // Sinn und Form. - 15. Jg., 1963. - S. 184-203.

Deutsche Lyrik von den Anfängen bis zur Gegenwart (DL) / Hrsg. von W. Killy. - In 10 Bd. - München : Deutscher

Taschenbuch, 2001.

Freund, W. Deutsche Lyrik: Interpretationen vom Barock bis zur Gegenwart / W. Freund. - 2. Aufl. - München :

Fink, 1990. - 224 S.

Gottsched, J. Ch. Versuch einer Critischen Dichtkunst / J. Ch. Gottsched. - Leipzig : Breitkopf, 1730. - 804 S.

Grünbein, D. Memorandum / D. Grünbein // Hildebrand O. Poetologische Lyrik von Klopstock bis Grünbein. Ge-

dichte und Interpretationen. - Köln ; Weimar ; Wien : Böhlau, 2003. - S. 324.

Hauptmann, G. Des großen Kampffliegers, Landfahrers, Gauklers und Magiers Till Eulenspiegel Abenteuer, Strei-

che, Gaukeleien, Gesichte und Träume / G. Hauptmann. - Berlin : Fischer, 1928. - 303 S.

Holthusen, H. E. Hier in der Zeit. - München : Piper, 1989. - 65 S.

Kayser, W. Geschichte des deutschen Verses. - 4. Aufl. - Tübingen : Francke, 1991. - 156 S.

Kayser, W. Kleine deutsche Versschule. - 26. Aufl. - Tübingen ; Basel : Francke, 1999. - 125 S.

Kelletat, A. Zum Problem der antiken Metren im Deutschen // Der Deutschunterricht. - 1964. - No 16. - S. 50-85.

Kleist, H. Werke in zwei Bänden / H. Kleist. - Bd. 1. - Berlin ; Weimar : Aufbau, 1983. - 348 S.

Knörrich, O. Lexikon lyrischer Formen / O. Knörrich. - 2. Aufl. - Stuttgart : Kröner, 2005. - 274 S.

Kolmar, G. Der Schwimmer / G. Kolmar // Weibliches Bildnis. Gedichte. - München : Köhl, 1980. - S. 254.

Lyriktheorie. Texte vom Barock bis zur Gegenwart / Hrsg. von L. Völker. - Stuttgart : Reclam, 1990. - 468 S.

Moennighoff, B. Metrik / B. Moennighoff. - Stuttgart : Reclam, 2004. - 113 S.

Mörike, E. Erzählungen und Gedichte / E. Mörike. - München ; Zürich : Knaur, 1965. - 320 S.

Müller, H. Geschichten von Homer / H. Müller. - URL: https://www.lyrikline.org/de/gedichte/geschichten-von-homer-3227 (mode of access: 20.03.2021). - Text : electronic.

Novalis, Fr. Werke in einem Band / Fr. Novalis. - Berlin ; Weimar : Aufbau, 1985. - 394 S.

Otfrieds Evangelienbuch / Hrsg. von Erdmann-Wolff. - Tübingen : Niemeyer, 1965. - 65 S.

Platen A. von: Werke in zwei Bänden. Band 1: Lyrik A. von Platen. - München : Saur, 1982. - 580 S.

Saar, F. L. A. Wiener Elegien / F. L. A. Saar. - URL: https://www.zgedichte.de/gedichte/ferdinand-ludwig-adamvon-saar/wiener-elegien.html (mode of access: 20.03.2021). - Text : electronic.

Suvin, D. Brechts Gedichtfassung des Kommunistischen Manifests / D. Suvin // Das Argument. - 2008. - No 282. -

S. 607-615.

Wagenknecht, Ch. Deutsche Metrik: Eine historische Einführung / Ch. Wagenknecht. - Beck, 1981. - 139 S.

Weinheber, J. Auf das Unabwendbare / J. Weinheber // Das Rowohlt Lesebuch der Poesie / Hrsg. von A. Praesent. Reinbek bei Hamburg : Rowohlt, 1983.

\section{References}

Arndt, E. (1984). Deutsche Verslehre. Ein Abriss. 9 ed. Berlin, Volk \& Wissen. 229 p.

Bantel, O. (1965). Grundbegriffe der Literatur. 4 ed. Frankfurt a. M., Hirschgraben. 103 p.

Behrmann, A. (1989). Einführung in den neueren deutschen Vers von Luther bis zur Gegenwart. Stuttgart, Metzler. 179 p.

Beissner, Fr. (1991). Geschichte der deutschen Elegie. Berlin, Gruyter. 240 p.

Braune-Ebbinghaus (Ed.). (1965). Althochdeutsches Lesebuch. Tübingen, Niemeyer. 187 p.

Breuer, D. (1999). Deutsche Metrik und Versgeschichte. 4 ed. München, Fink. 414 p.

Bunge, H.-J. (1963). Das Manifest von Bertolt Brecht. In Sinn und Form. 15. Jg., pp. 184-203.

Erdmann-Wolff (Ed). (1965). Otfrieds Evangelienbuch. Tübingen, Niemeyer. 65 S.

Freund, W. (1990). Deutsche Lyrik. Interpretationen vom Barock bis zur Gegenwart. 2 ed. München, Fink. 224 p. 303 p.

Gasparov, M. L. (1989). Ocherk istorii evropeiskogo stikha [Essay on the History of European Verse]. Moscow, Nauka.

Gasparov, M. L. (1999). Metr i smysl. Ob odnom iz mekhanizmov kulturnoy pamyati [Meter and Meaning. About One of the Mechanisms of Cultural Memory]. Moscow, RGGU. 289 p.

Gottsched, J. Ch. (1730). Versuch einer Critischen Dichtkunst. Leipzig, Breitkopf. 804 p.

Grünbein, D. (2003). Memorandum. In Hildebrand, O. (Ed.). Poetologische Lyrik von Klopstock bis Grünbein. Gedichte und Interpretationen. Köln, Weimar, Wien, Böhlau, p. 354.

Hauptmann, G. (1928). Des großen Kampffliegers, Landfahrers, Gauklers und Magiers Till Eulenspiegel Abenteuer, Streiche, Gaukeleien, Gesichte und Träume. Berlin, Fischer. 303 p.

Holthusen, H. E. (1989). Hier in der Zeit. München, Piper. 65 p.

Kayser, W. (1991). Geschichte des deutschen Verses. 4 ed. Tübingen, Francke. 156 p.

Kayser, W. (1999). Kleine deutsche Versschule. 26 ed. Tübingen, Basel, Francke. 125 p.

Kelletat, A. (1964). Zum Problem der antiken Metren im Deutschen. In Der Deutschunterricht. No. 16, pp. 50-85.

Kholshevnikov, V. E. (2002). Osnovy stikhovedeniya. Russkoe stikhoslozhenie [Fundamentals of Poetry. Russian Versification]. Saint Petersburg, Akademiya. 208 p. buch.

Killy, W. (Ed.). (2001). Deutsche Lyrik von den Anfängen bis zur Gegenwart (DL), in 10 vols. München, Deutscher Taschen- 
Kleist, H. (1983). Werke in zwei Bänden. Vol. 1. Berlin, Weimar, Aufbau. 348 p.

Knörrich, O. (2005). Lexikon lyrischer Formen. 2 ed. Stuttgart, Kröner. 274 p.

Kolmar, G. (1980). Der Schwimmer. In Weibliches Bildnis. Gedichte. München, Köhl, p. 254.

Moennighoff, B. (2004). Metrik. Stuttgart, Reclam. 113 p.

Mörike, E. (1965). Erzählungen und Gedichte. München, Zürich, Knaur. 320 p.

Müller, H. Geschichten von Homer. URL: https://www.lyrikline.org/de/gedichte/geschichten-von-homer-3227 (mode of access: 20.03 .2021$)$.

Novalis, Fr. (1985). Werke in einem Band. Berlin, Weimar, Aufbau. 394 p.

Platen, A. von. (1982). Werke in zwei Bänden. Vol. 1: Lyrik A. von Platen. München, Saur. 580 S.

Saar, F. L. A. Wiener Elegien. URL: https://www.zgedichte.de/gedichte/ferdinand-ludwig-adam-von-saar/wiener-elegien. html (mode of access: 20.03.2021).

Suvin, D. (2008). Brechts Gedichtfassung des Kommunistischen Manifests. In Das Argument. No. 282, pp. 607-615.

Tomashevsky, B. V. (2001). Teoriya literatury. Poetika [Theory of Literature. Poetics]. Moscow, Aspekt Press. 334 p.

Völker, L. (Ed.). (1990). Lyriktheorie. Texte vom Barock bis zur Gegenwart. Stuttgart, Reclam. 468 p.

Wagenknecht, Ch. (1981). Deutsche Metrik. Eine historische Einführung. Beck. 139 p.

Weinheber, J. (1983). Auf das Unabwendbare. In Praesent, A. (Ed.). Das Rowohlt Lesebuch der Poesie. Reinbek bei Hamburg, Rowohlt.

\section{Данные об авторе}

Андреюшкина Татьяна Николаевна - доктор филологических наук, доцент, профессор кафедры теории и практики перевода, Тольяттинский государственный университет (Тольятти, Россия).

Адрес: 445020, Россия, г. Тольятти, ул. Белорусская, 14.

E-mail: andrejushkina@tltsu.ru.

\section{Author's information}

Andreiushkina Tatiana Nikolaevna - Doctor of Philology, Associate Professor, Professor of Department of Theory and Practice of Translation, Togliatti State University (Togliatti, Russia). 